\title{
Dual role of the mitochondrial protein frataxin in astrocytic tumors
}

\author{
Elmar Kirches ${ }^{1}$, Nadine Andrae ${ }^{1}$, Aline Hoefer ${ }^{2}$, Barbara Kehler ${ }^{1,3}$, Kim Zarse ${ }^{4}$, Martin Leverkus ${ }^{3}$, Gerburg Keilhoff ${ }^{5}$, \\ Peter Schonfeld ${ }^{5}$, Thomas Schneider ${ }^{6}$, Annette Wilisch-Neumann ${ }^{1}$ and Christian Mawrin ${ }^{1}$
}

The mitochondrial protein frataxin (FXN) is known to be involved in mitochondrial iron homeostasis and iron-sulfur cluster biogenesis. It is discussed to modulate function of the electron transport chain and production of reactive oxygen species (ROS). FXN loss in neurons and heart muscle cells causes an autosomal-dominant mitochondrial disorder, Friedreich's ataxia. Recently, tumor induction after targeted FXN deletion in liver and reversal of the tumorigenic phenotype of colonic carcinoma cells following FXN overexpression were described in the literature, suggesting a tumor suppressor function. We hypothesized that a partial reversal of the malignant phenotype of glioma cells should occur after FXN transfection, if the mitochondrial protein has tumor suppressor functions in these brain tumors. In astrocytic brain tumors and tumor cell lines, we observed reduced FXN levels compared with non-neoplastic astrocytes.

Mitochondrial content (citrate synthase activity) was not significantly altered in U87MG glioblastoma cells stably overexpressing FXN (U87-FXN). Surprisingly, U87-FXN cells exhibited increased cytoplasmic ROS levels, although mitochondrial ROS release was attenuated by FXN, as expected. Higher cytoplasmic ROS levels corresponded to reduced activities of glutathione peroxidase and catalase, and lower glutathione content. The defect of antioxidative capacity resulted in increased susceptibility of U87-FXN cells against oxidative stress induced by $\mathrm{H}_{2} \mathrm{O}_{2}$ or buthionine sulfoximine. These characteristics may explain a higher sensitivity toward staurosporine and alkylating drugs, at least in part. On the other hand, U87-FXN cells exhibited enhanced growth rates in vitro under growth factor-restricted and hypoxic conditions and in vivo using tumor xenografts in nude mice. These data contrast to a general tumor suppressor function of FXN but suggest a dual, pro-proliferative but chemosensitizing role in astrocytic tumors. Laboratory Investigation (2011) 91, 1766-1776; doi:10.1038/labinvest.2011.130; published online 22 August 2011

KEYWORDS: chemotherapy; frataxin; glioma; oxidative stress

A GAA repeat expansion in the frataxin gene causes the lethal disorder Friedreich's ataxia (FRDA), which is characterized by degeneration of spinocerebellar tracts, dorsal columns and pyramidal tracts, as well as heart failure. The mutation abolishes gene transcription, leading to a loss of the mitochondrial protein frataxin (FXN), mitochondrial iron accumulation and oxidative stress. ${ }^{1,2}$ Knockout of the FXN homolog Yfhlp in yeasts impairs respiration and causes mitochondrial DNA depletion and iron accumulation., Defects of iron-sulfur proteins in myocardial biopsies of FRDA patients confirmed a defective iron-sulfur cluster (ISC) biogenesis in the Yfhlp knockout model. ${ }^{5}$ According to the current view, mammalian FXN is directly involved in ISC biogenesis, ${ }^{6}$ the central steps of which take place in the mitochondrial matrix. FXN oligomers exhibit large capacity for binding ferrous iron. After binding of FXN to the ISCscaffold protein, iron may be directly handed over to the metal-free ISCs, which are then transferred to their final targets. ${ }^{7}$ ISC proteins include complexes of the electron transport chain (ETC) or ferrochelatase, an enzyme of heme biogenesis. ${ }^{8}$ FXN is thus essential for the function of ISCand heme-containing ETC complexes and of cytochrome c.

In tumor cells, FXN has been discussed as a modulator of energy metabolism, with an impact on growth characteristics. FXN-transformed colon cancer cell lines exhibited enhanced respiration and were characterized by decreased growth rates

\footnotetext{
Department of Neuropathology, Otto-von-Guericke University, Magdeburg, Germany; ${ }^{2}$ Department of Neuropathology, Friedrich-Schiller University, Jena, Germany; ${ }^{3}$ Department of Experimental Dermatology, Otto-von-Guericke University, Magdeburg, Germany; ${ }^{4}$ Department of Human Nutrition, Friedrich-Schiller University, Jena, Germany; ${ }^{5}$ Department of Biochemistry and Cell Biology, Otto-von-Guericke University, Magdeburg, Germany and ${ }^{6}$ Department of Neurosurgery, Otto-von-Guericke University, Magdeburg, Germany

Correspondence: Dr C Mawrin, MD, Department of Neuropathology, Otto-von-Guericke-University, Leipziger Strasse 44, D-39120 Magdeburg, Germany.

E-mail: christian.mawrin@med.ovgu.de

Received 27 January 2011; revised 04 July 2011; accepted 13 July 2011
} 
in soft agar assays and nude mice. ${ }^{9}$ These results were interpreted in terms of cancer growth inhibition by antioxidative properties of FXN, leading to an enhancement of oxidative phosphorylation (OXPHOS). The reciprocal experiment was a hepatocyte-specific FXN knockout in mice. The induction of multiple hepatic tumors with decreased mitochondrial metabolism and iron-sulfur proteins suggested a tumor suppressor function for FXN. ${ }^{10}$

Malignant astrocytic tumors are among the most frequent intracranial neoplasms. Despite recent advances of chemotherapy, the improvement of median overall survival of patients with glioblastoma (GBM) remains a major challenge. ${ }^{11}$ In this report, we investigated the role of FXN in astrocytic tumors and GBM cell lines. We hypothesized that the malignant biological behavior of astrocytic tumor cells, especially regarding tumor growth, should be attenuated by FXN transfection in vitro and in vivo, if the protein has tumor suppressor properties in these cells. If these biological effects were connected with the suggested antioxidative and OXPHOS-protecting properties of FXN, transfected cells should exert lower reactive oxygen species (ROS) generation and higher aconitase activity. In addition, it is likely that the transfected cells are less sensitive to externally applied oxidative stress.

\section{MATERIALS AND METHODS Vector Construction}

pCl-neo-FXN was generated by cloning a hemagglutintagged human frataxin cDNA into pCl-neo (Promega, Mannheim, Germany).

\section{Cell Culture}

The GBM cell lines U87MG, U118MG, U138MG and LN405 were purchased from the American Type Culture Collection (ATCC) or the German Collection of Microorganisms and Cell Cultures, respectively. Transformed cell lines U87-FXN and U87-Neo were generated by lipofectamine-2000 transfection of the plasmids into U87MG cells and culturing under $0.2 \mathrm{mg} / \mathrm{ml}$ G418. This concentration had been determined as the minimal concentration, which kills parental cells within 2 weeks. Developing colonies of resistant cells were selectively transferred to new flask and maintained under G418. The cell lines used in the present study thus represent cell clones. Human cortical astrocytes were purchased from ScienCell Research Laboratories (Carlsbad, CA) and embryonal rat astrocytes prepared in our laboratory. ${ }^{12}$ All cells were cultured in DMEM with $10 \%$ FCS. The identity of the cell lines was analyzed using the AmpFSTR kit and the software GeneMapper ID v3.2 (Applied Biosystems), allowing automatic identification of alleles of 11 polymorphic chromosomal markers and amelogenin by fluorescent multiplex PCR. The transgenic lines U87-FXN and U87-Neo showed patterns, which were identical for all markers analyzed, proving these cell lines to be derivatives of U87MG cells, which had been originally obtained from the
ATCC. Three of these markers, amelogenin (X), vWA (alleles 15,17 ) and THO1 (allele 9.3) are published for the parental line U87MG in the ATCC database (http://www.ATCC.org). OLN-93 was originally derived from a primary rat brain glial culture and resembles primary oligodendrocytes. It was a kind gift of Professor Richter-Landsberg (Department of Biology, University of Oldenburg, Germany), who first described the line.

\section{Human Tumor Tissues}

The frozen samples were taken from the files of the Department of Neuropathology of the Otto-von-Guericke University. The samples had been obtained during routine neurosurgical excision of samples for diagnostic purposes. Diagnosis had been performed by an experienced neuropathologist $(\mathrm{CM})$.

\section{Mixed Rat Brain Cell Cultures}

Neuronal/glial mixed cultures were prepared from cerebellum of 4-day-old rats in DMEM with $10 \%$ FCS and $1 \%(\mathrm{v} / \mathrm{v})$ penicillin/streptomycin. After mechanical dispersion, aliquots of the suspension were plated in poly-D-lysine coated dishes at a final density of $2 \times 10^{6}$ cells per $8 \mathrm{~cm}^{2}$. After $48 \mathrm{~h}$, non-adherent cells were removed by medium change. After 10 days, the cells were used for experiments. For serum/glucose deprivation ( $S G D)$, growth medium was replaced by serum- and glucose-free DMEM for $24 \mathrm{~h}$.

\section{Qualitative and Real-Time PCR}

RNA was isolated using the RNeasy-mini-kits from Qiagen (Hilden, Germany). FXN-PCR from cDNA was performed with the primers TAAGCGTTATGACTGGACTGG and GAA TAGGCCAAGGAAGACAAG, $\beta$-actin (ACC ACC CCA GCC ATG TAC G and ATG TCA CGC ACG ATT TCC C) serving as a reference. For SYBR-green-based Real-Time PCR on an ABI-7000-SDS (Applied Biosystems, CA), the same primers were used with identical annealing temperature $\left(57^{\circ} \mathrm{C}\right)$.

\section{Western Blotting}

Cells were washed twice with ice-cold PBS and harvested by scraping in RIPA buffer (10 mM Tris- $\mathrm{HCl}, \mathrm{pH} 7.4 ; 150 \mathrm{mM}$ $\mathrm{NaCl} ; 50 \mathrm{mM} \mathrm{NaF} ; 1 \mathrm{mM}$ EDTA; $1 \%$ Triton X-100; $0.1 \%$ SDS; $0.5 \%$ deoxycholate) with DTT, Na-vanadate and protease inhibitors. In all, $40 \mu \mathrm{g}$ protein was separated by SDSPAGE and transferred onto nitrocellulose membranes. After blocking, membranes were incubated with primary antibodies in TBST overnight at $4{ }^{\circ} \mathrm{C}$, followed by chemiluminescence (Millipore, Schwalbach, Germany). Primary antibodies were directed against frataxin (Mitosciences, 1:300) and $\beta$-actin (SIGMA, 1:1000).

\section{Immunofluorescence}

Cultures were washed twice with PBS, fixed 30 min using $4 \%$ paraformaldehyde in PBS. After a blocking step with $1 \%$ goat 
serum for $30 \mathrm{~min}$, cells were incubated for $3 \mathrm{~h}$ at room temperature with antibodies in PBS with $0.3 \%$ Triton X-100 and $1 \%$ goat serum: rabbit anti-frataxin $(\mathrm{H}-155$, Santa Cruz Biotechnology, Santa Cruz, 1:500) in combination with mouse anti-GFAP (Chemicon, Temecula, 1:1000), mouse anti-MAP2 (Chemicon, 1:2500), mouse anti-NG2 (Chemicon, 1:500), anti-mouse S100b (Chemicon, 1:100) or a rat biotin-conjugated anti-Isolectin B4 antibody (Vector Laboratories Inc., Burlingame, CA; 1:100). Specificity of immunoreactions was controlled by application of buffer instead of primary antibodies, which never resulted in immunostaining. Cultures were washed in PBS $(3 \times 5 \mathrm{~min})$ and incubated for $3 \mathrm{~h}$ with a combination of secondary antibodies (Molecular Probes, Göttingen, Germany; 1:500): goat anti-rabbit-IgG Alexa Fluor 546/goat anti-mouse-IgG Alexa Fluor 488. Since the IB4 antibody is biotin conjugated, here the secondary antibody step included only the use of goat anti-rabbit-IgG Alexa Fluor 546.

\section{MTT, Crystal Violet and BrdU Assays}

To evaluate the surviving cell fraction, 3-(4,5-dimethylthiazol-2-yl)-2,5-diphenyltetrazolium bromide (MTT) or crystal violet assays were performed after treatment of cells in 96well microplates as indicated in the figures. Cells were stained for $2 \mathrm{~h}$ with $0.75 \mathrm{mg} / \mathrm{ml} \mathrm{MTT}$ salt at $37^{\circ} \mathrm{C}$, lysed in $100 \mu \mathrm{l}$ dimethyl sulfoxide and absorbance was read at $562 \mathrm{~nm}$. For determination of hypoxia sensitivity, equal amounts of cells were seeded into cell culture flasks and incubated for 24 or $48 \mathrm{~h}$ (as indicated in Figure 5) either in the usual incubator under normoxic conditions (control), or in an $\mathrm{N}_{2}$-fumigated hypoxia chamber with $5 \% \mathrm{CO}_{2}$, but only $0.1 \%$ oxygen. MTT assays were performed immediately after the incubation period, but under normoxic conditions. Signals thus represent the number of cells present, but not differences in mitochondrial oxygen saturation. For crystal violet assays, cells were stained with $50 \mu \mathrm{l}$ of $0.5 \%$ crystal violet and $20 \%$ methanol for $15 \mathrm{~min}$. After washing four times, the dried wells were incubated $20 \mathrm{~min}$ with $200 \mu \mathrm{l}$ methanol and measured at $595 \mathrm{~nm}$. The percentage of viable cells was defined as optical density relative to untreated controls. ${ }^{13}$ For the determination of cell proliferation (DNA synthesis), the BrdU-based assay from Roche (Mannheim) was used.

\section{Enzyme and GSH Assays}

Activities of glutathione peroxidase (GPx) and catalase (CAT) and total glutathione were determined in fresh cell lysates generated by sonication of cells in PBS using the GPx-340 kit from Oxis Research (Foster City, CA), the catalase assay kit from Calbiochem (Darmstadt, Germany) and the GSH assay kit from Sigma (Taufkirchen, Germany). Aconitase activity was measured with a reaction mixture containing $60 \mathrm{mM}$ sodium citrate, $0.4 \mathrm{mM} \mathrm{NADP}+, 0.6 \mathrm{mM} \mathrm{MnCl} 2$ and $4 \mathrm{U} / \mathrm{ml}$ isocitrate dehydrogenase dissolved in $50 \mathrm{mM}$ Tris- $\mathrm{HCl}, \mathrm{pH}$ 7.4. Absorbance was read at $340 \mathrm{~nm}$ in the plate reader.
The assay for citrate synthase (CS), an established mitochondrial marker enzyme, is based on the CS-mediated reaction of acetyl-CoA with oxaloacetate to citrate and free coenzyme A. Coenzyme A reacts with Ellman's reagent (5,5'-dithiobis(2-nitrobenzoic acid)) to 2-nitro-5-thiobenzoic acid, which is quantified by absorbance at $412 \mathrm{~nm}$.

\section{Fluorimetric DCF Assay}

Cells were resuspended in PBS with $20 \mathrm{mM}$ glucose at a density of 20000 cells $/ \mathrm{ml}$ and immediately loaded with $2 \mu \mathrm{M}$ DCFH-diacetate for $15 \mathrm{~min}$ at $37^{\circ} \mathrm{C}$. DCFH-DA becomes hydrolyzed to DCF, a fluorescent probe for ROS detection (excitation at $458 \mathrm{~nm}$ and emission at $520 \mathrm{~nm}$ ), allowing time-dependent monitoring of intracellular ROS generation at $25^{\circ} \mathrm{C}$.

\section{Isolation of Mitochondria}

To isolate mitochondria from cells, $2 \times 10^{7}$ cells were suspended in $0.8 \mathrm{ml}$ of ice-cold sucrose-based medium $(250 \mathrm{mM}$ sucrose, $5 \mathrm{mM}$ Hepes, $0.1 \mathrm{mM}$ EDTA, pH 7.2) and homogenized using a Dounce homogenizer. After the addition of $0.8 \mathrm{ml}$ sucrose-based medium, the mixture was centrifuged $\left(700 \mathrm{~g}, 10 \mathrm{~min}\right.$ at $\left.4{ }^{\circ} \mathrm{C}\right)$. The resulting supernatant was again centrifuged $\left(12000 \mathrm{~g}, 15 \mathrm{~min}\right.$ at $\left.4^{\circ} \mathrm{C}\right)$. The mitochondrial pellet was washed once in sucrose-based medium and resuspended in $0.5 \mathrm{ml}$ of the same medium. The protein content of this stock suspension was measured by BioRad Protein Kit.

\section{Mitochondrial ROS Generation}

Mitochondrial ROS generation was estimated as release of $\mathrm{H}_{2} \mathrm{O}_{2}$ using the Amplex Red (AR)/horse radish peroxidase (HRP) system $\left(37^{\circ} \mathrm{C}\right)$. Briefly, mitochondria $(0.4 \mathrm{mg}$ protein/ $\mathrm{ml})$ were incubated in medium supplemented with $5 \mu \mathrm{M} \mathrm{AR}$ plus HRP ( 2 units/ml) to detect $\mathrm{H}_{2} \mathrm{O}_{2}$ release. The nonfluorescent $\mathrm{AR}$ becomes oxidized by $\mathrm{H}_{2} \mathrm{O}_{2}$ to the fluorescent resorufin. The fluorescence of the latter compound was fluorimetrically monitored at $37^{\circ} \mathrm{C}$ using a Perkin-Elmer Life Sciences LS-50B fluorescence spectrometer (excitation at $560 \mathrm{~nm}$ and emission at $590 \mathrm{~nm}$ ). The incubation medium contained $110 \mathrm{mM}$ mannitol, $60 \mathrm{mM} \mathrm{KCl}, 60 \mathrm{mM}$ Tris- $\mathrm{HCl}$, $10 \mathrm{mM} \mathrm{KH}_{2} \mathrm{PO}_{4}, 0.5 \mathrm{mM}$ EGTA (pH 7.4) and $5 \mathrm{mM}$ pyruvate plus $5 \mathrm{mM}$ malate. Changes in the resorufin fluorescence were calibrated with $\mathrm{H}_{2} \mathrm{O}_{2}$.

\section{Tumor Growth in Nude Mice}

In all, $3 \times 10^{5}$ matrigel-embedded tumor cells were subcutaneously injected into the flanks of Swiss nude (Crl:Nu (Ico)-Fox1) mice (Charles River Laboratories, Germany). Animal experimentation was conducted according to the local Ethics Committee approvals. During a period of 6 weeks, the tumor volumes were estimated by external measurement of length and width and calculated according to the formula $\left(x y^{2}\right) / 2, x>y$. After 6 weeks, the tumors were removed from the killed animals and tumor weight and total 
GSH content were determined. Tumor histology was analyzed in routine H\&E slices by a neuropathologist (CM).

\section{Statistics}

Multiple comparisons were performed with an ANOVA, followed by Tukey post hoc test. A two-sided $t$-test was used to evaluate experiments comparing only two treatments or cell lines. Significance was assumed for $P \leq 0.05$. All calculations were performed with the software package SPSS, version 14.0.

\section{RESULTS}

Frataxin Expression in Glial Cells and Astrocytic Tumors Although FXN is detectable in oligodendrocytes ${ }^{14}$ and astrocytes ${ }^{15}$ glial tumors had not been analyzed so far. As shown in Figure 1a, unstimulated mixed cell cultures (A-E) displayed FXN expression in neuronally differentiated cells (A), oligodendrocytes (C), precursor cells (D), and in remarkable amounts in microglial cells $(\mathrm{E})$, while it was only sparsely detected in astrocytes (B). SGD (F-J) resulted in increased FXN expression especially in astrocytes (G). The latter experiment was performed three times independently and stains in three culture dishes were performed per experiment. In all nine dishes, visual inspection revealed a higher fraction of FXN/GFAP double-stained cells, although no quantitative measurement of fluorescence levels was applied. Micrograph (K) is a higher magnification of $(G)$, while $\mathrm{L}$ and $\mathrm{M}$ represent two other independent experiments. The micrographs of higher magnification better show the dot-like, mitochondrial FXN staining (red) in GFAP-positive (green) astrocytes. These data suggested that FXN expression is low in resting astrocytes, but induced by astroglial activation due to cellular stress. Since astrocytes are the most common cell type giving rise to intracranial neoplasms, we wondered whether FXN has a role in astrocytic tumors.

FXN mRNA was present in all tumors studied (Figure 1b) and a reduction appears to occur in high-grade tumors (Figure 1c). Western blot studies revealed two bands of low molecular weight, which can be interpreted in terms of a two-step processing of the cytoplasmic precursor by proteases within the mitochondria, ${ }^{16,17}$ the smaller protein representing mature FXN. A marked expression of the latter in astrocytes was strongly reduced in astrocytic tumors $(P<0.05)$ according to densitometrical scans of western blots (Figure 1d) and in tumor cell lines (Figure 1e).

\section{Impact of FXN on Mitochondrial Metabolism and Oxidative Stress in Human Glioma Cells}

We next asked whether re-expression of FXN might have beneficial effects on oxidative mitochondrial metabolism in GBM cells and hence reverse tumorigenic phenotype. ${ }^{9} \mathrm{We}$ stably transfected U87MG cells with human wild-type FXN under control of a constitutive promoter (CMV), further referred as U87-FXN.

Immunofluorescence studies confirmed the mitochondrial localization of FXN in normal rat astrocytes (Figure 2a, A).
Although some degree of dot-like FXN staining occurred in a few parental U87MG cells (B), perinuclear co-staining of FXN and mitotracker was clearly enhanced in U87-FXN compared with U87-Neo cells. Nearly all U87-FXN cells showed strong FXN expression (C and D). Western blotting demonstrated a massive induction of the two mitochondrial FXN proteins of low molecular weight (Figure 2b).

Due to the known function of FXN in iron homeostasis, we analyzed protein levels of ferritin, transferrin and the ISCscaffold protein by western blot, but did not detect substantial changes (data not shown).

Following the working hypothesis that FXN overexpression in cancer cells may activate oxidative mitochondrial metabolism and in addition diminish respiratory chain-linked ROS generation, ${ }^{9}$ we first analyzed CS activity as a measure of total mitochondrial content. The slopes of protein normalized enzyme activities were $(1.34 \pm 0.016) \times 10^{-3} / \mathrm{min} / \mu \mathrm{g}$ for U87-FXN and $(1.18 \pm 0.017) \times 10^{-3} \mathrm{~min} / \mu \mathrm{g}$ for U87-Neo $(n=6)$. This result excluded a major impact of FXN overexpression on total mitochondrial content, which thus should not have any impact on total ROS production of the cells. Moreover, CS activity was determined in four independent experiments in mitochondria isolated from four cell culture passages. The resulting activities normalized to mitochondrial protein $(6000 \pm 2980 \mathrm{nmol} / \mathrm{min} /$ $\mathrm{mg}$ in U87-FXN cells and $5900 \pm 2500 \mathrm{nmol} / \mathrm{min} / \mathrm{mg}$ in U87-Neo cells) revealed no major difference between the two clones.

The activity of the Krebs cycle enzyme aconitase (ACO) was now determined from whole cell extracts, since it essentially needs FXN to maintain its ISC content and is highly vulnerable to intra-mitochondrial ROS. ${ }^{18}$ Aconitase activity was about $26 \%$ increased in U87-FXN cells $(P<0.05$; Figure 3a). We also measured aconitase activity in mitochondrial preparations of both cell clones. Three independent measurements (three passages) all seemed to support a higher activity in the FXN transfected cells, although the difference did not become statistically significant due to large variation in absolute activities between the three sets of experiments (data not shown).

If this effect should rely on diminished ROS levels generated by the ETC, lower levels of DCF fluorescence would be expected in U87-FXN cells. Superoxide is released to both sides of the inner membrane and is converted to $\mathrm{H}_{2} \mathrm{O}_{2}$ by superoxide dismutases in cytoplasm ( $\mathrm{Cu} / \mathrm{Zn}-\mathrm{SOD})$ and matrix space (MnSOD). Hydrogen peroxide in the matrix, which is not inactivated by GPx, escapes mitochondria, enhancing the cytoplasmic DCF fluorescence of cells loaded with DCFH-DA. Surprisingly, the fluorescence was clearly enhanced in U87-FXN cells as compared with U87-Neo and U87MG cells (Figure 3b). These data were confirmed by corresponding differences in Amplex Red fluorescence, a measure of ROS release from cells into the medium (data not shown). In order to assess more specifically mitochondrial ROS generation in the absence of cytoplasmic ROS sources 
a unstressed
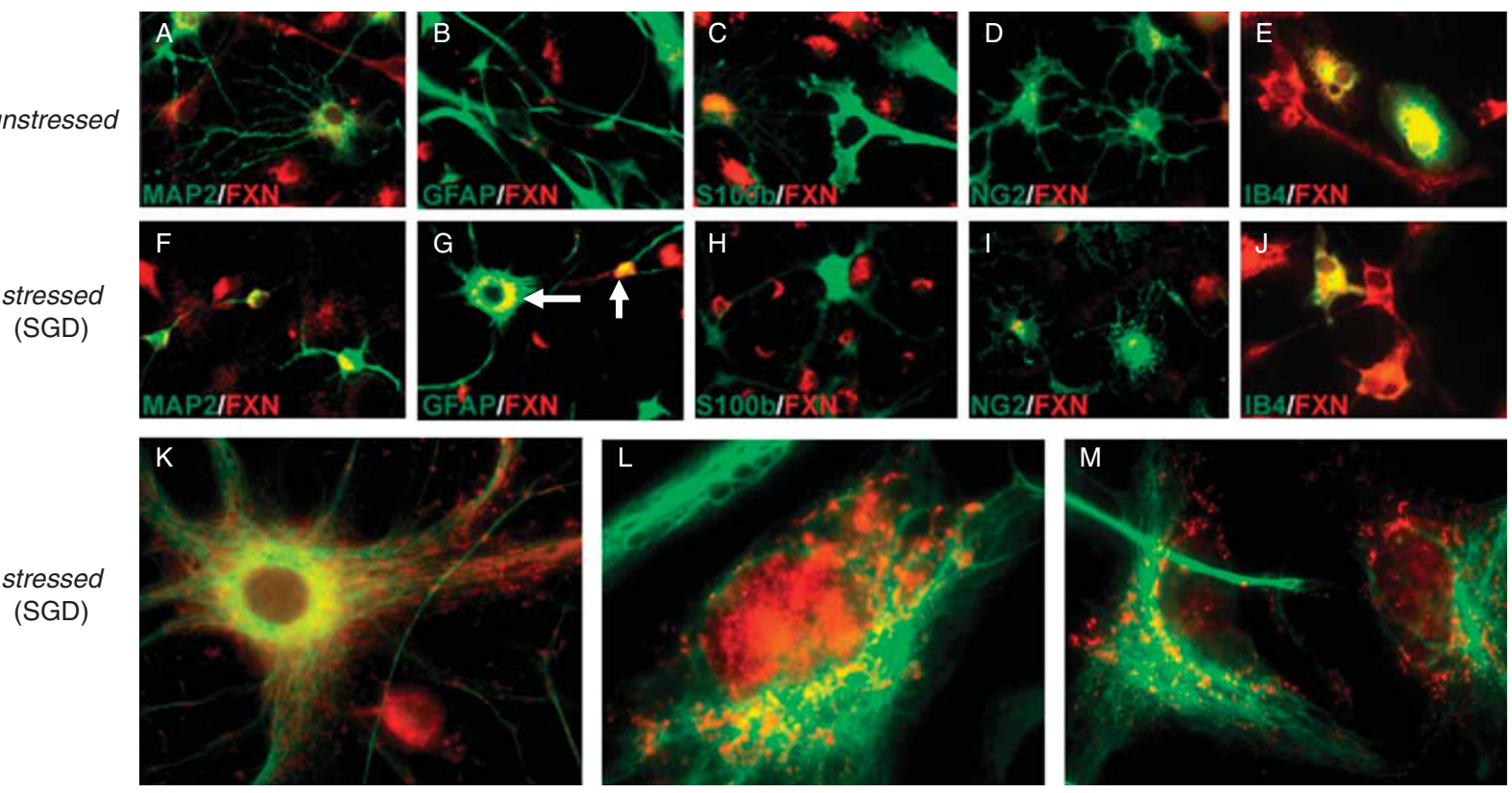

b

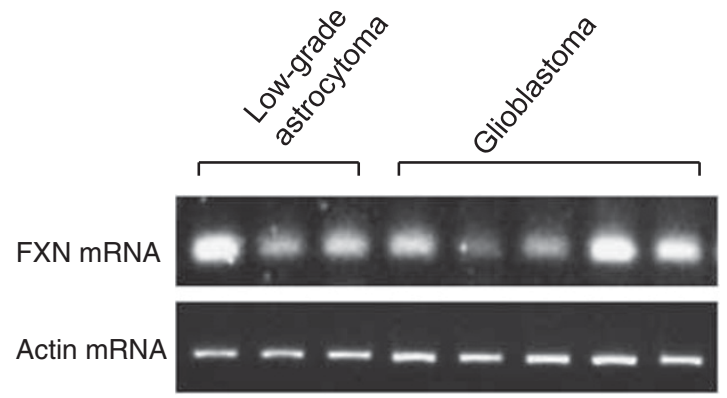

d

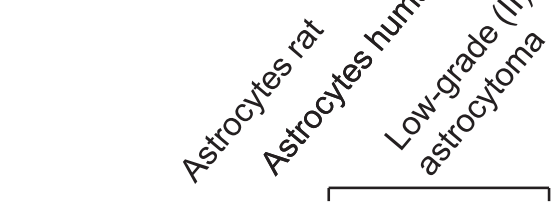

Int-FXN

Mat-FXN
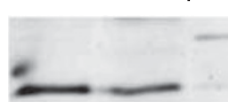

Actin
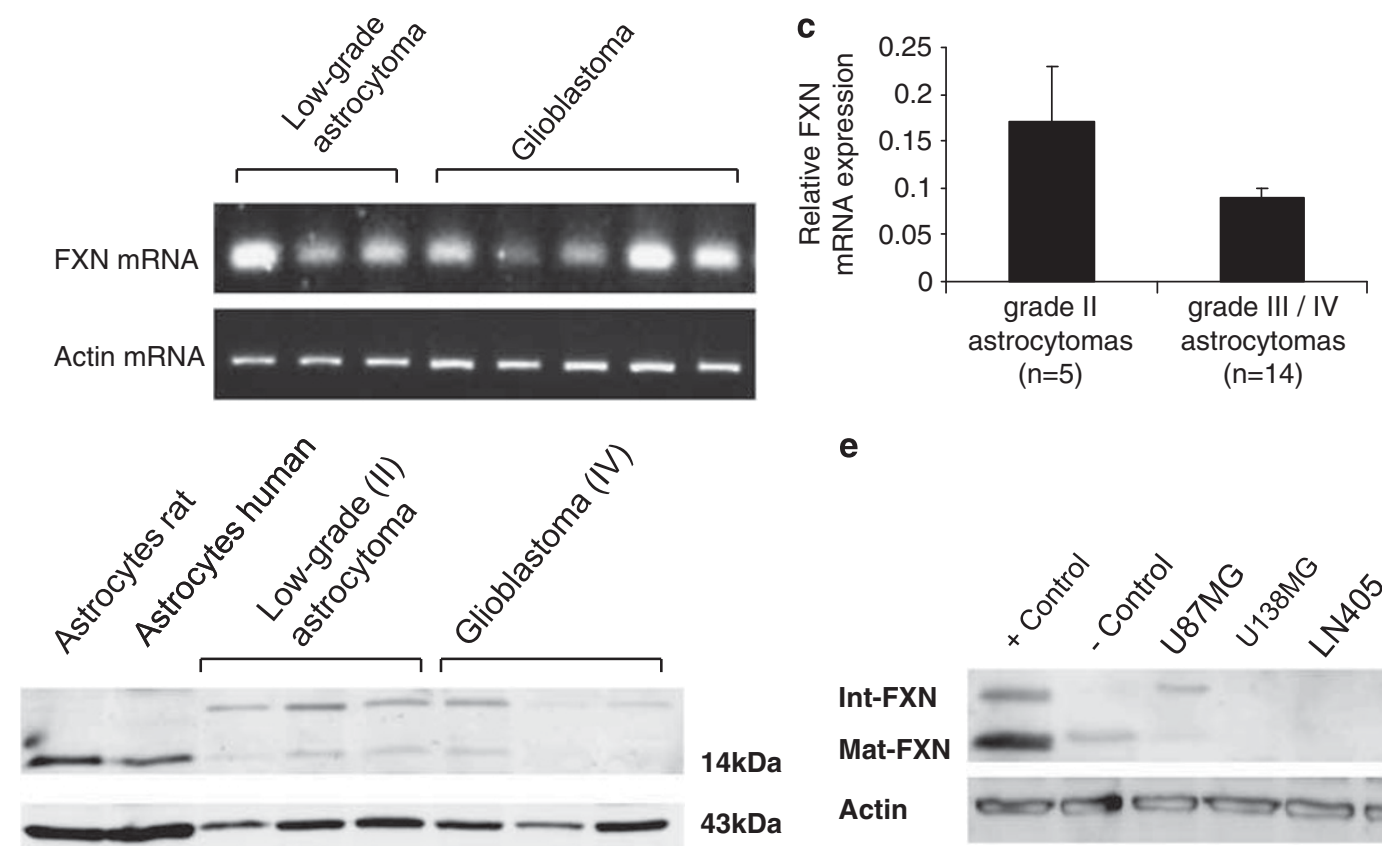

e

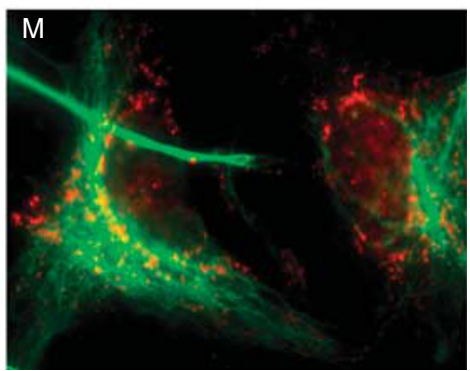

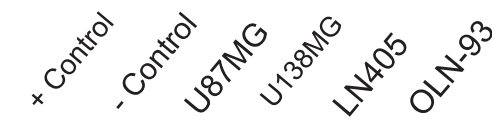

Int-FXN
Mat-FXN

43k Actin

$14 \mathrm{kDa}$

$43 \mathrm{kDa}$

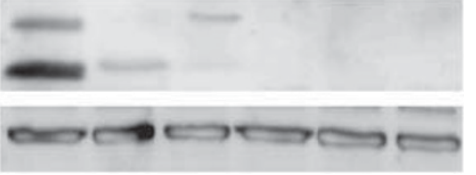

Figure 1 Expression of Frataxin (FXN) in glial cells and gliomas. (a) A mixed cell culture of rat cerebellum displays FXN expression in neuronal $(A)$, oligodendroglial $(C)$, precursor $(D)$ and microglial cells $(E)$, while astrocytes (B) are largely devoid of FXN staining. Following induction of cellular stress by serum/glucose deprivation (SGD), additionally this cell culture system shows increased FXN expression in astrocytes (arrows in G), while in other cell types FXN expression appears nearly unchanged $(\mathrm{F}, \mathrm{H}-\mathrm{J})$. (K-M) Show examples for the dot-like appearance of FXN staining in higher magnification. FXN mRNA is also expressed in human glial tumors of astrocytic origin (b), with quantitative reduction in high-grade malignant astrocytic tumors of WHO grades III and IV (anaplastic astrocytoma and glioblastoma) compared with diffuse astrocytomas of WHO grade II (c). (d) Protein levels of FXN are reduced in astrocytomas. (e) In astrocytic tumor cell lines, FXN protein is only faintly detectable in U87MG cells but appears to be missing in U138MG and LN405 cells. The oligodendrocytic line OLN-93 does not contain FXN. Positive and negative controls are colon carcinoma cells stably transfected with FXN or control vector as reported in Thierbach et al. ${ }^{10}$

and cytoplasmic antioxidant defences, we next measured Amplex Red fluorescence in mitochondrial suspensions (Figure 3c). The lower ROS release of suspensions of mitochondria isolated from FXN cells is in line with the increased ACO activity. The higher cytoplasmic ROS levels suggested either non-mitochondrial ROS sources and/or cytoplasmic antioxidant defences to be altered in the FXN transfected cells. 

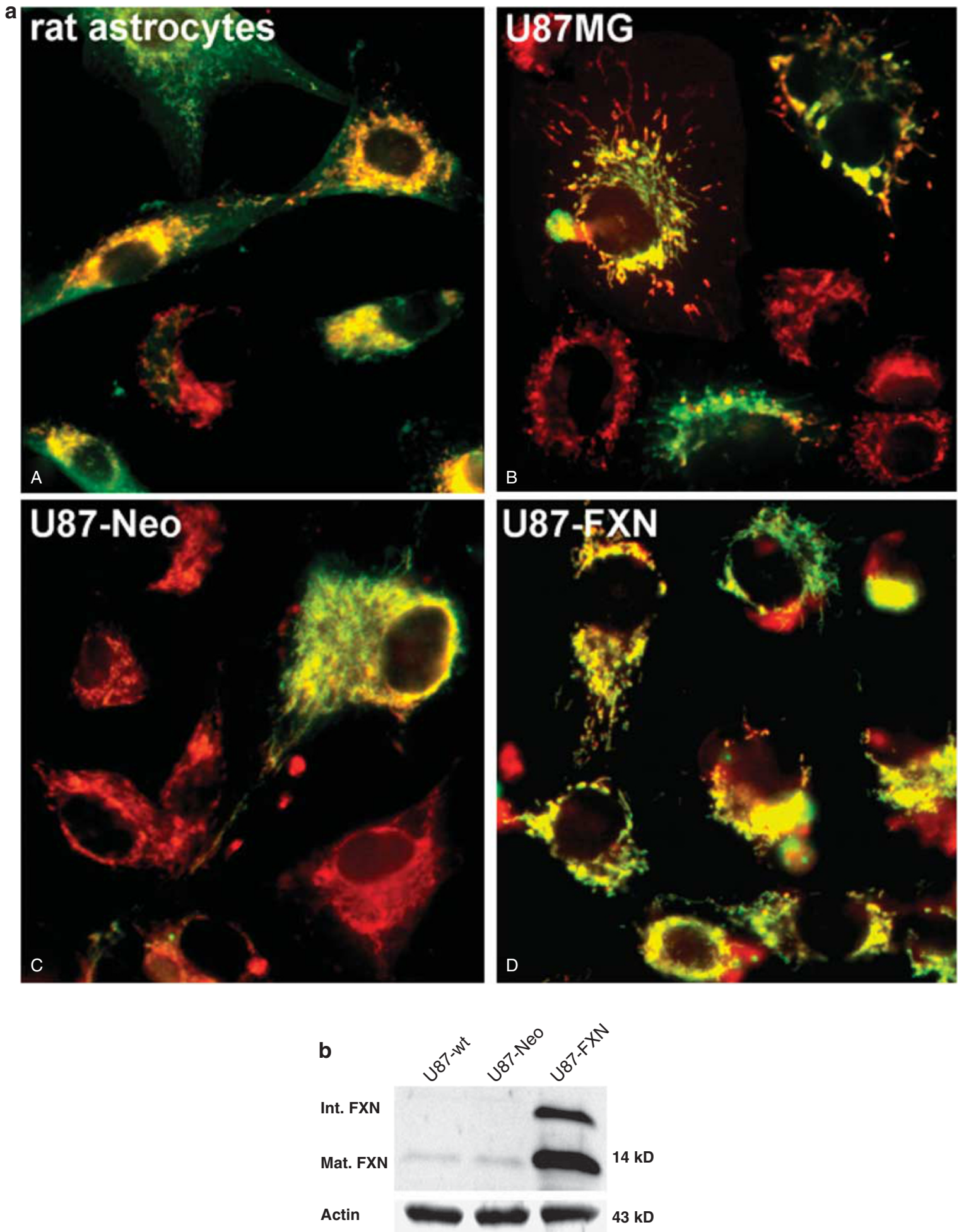

Figure 2 Generation of U87MG cells with stable overexpression of FXN. (a) Double-labeling studies using an anti-FXN antibody (green) and mitotracker (red) reveals the presence of FXN in few rat astrocytes (A), wild-type U87MG cells (B) and U87-Neo cells stably transfected with a control vector (C). In contrast, U87-FXN cells stably overexpressing frataxin (D) show a strong FXN signal with colocalization to mitochondria in nearly all cells. (b) Western blots showing intermediate and mature frataxin in U87-FXN cells (FXN), while the parental line U87MG (wt) and the mock-transfected line (U87-Neo) showed only minimal expression when the blots were incubated with a FXN antibody. 
$\mathbf{a}$
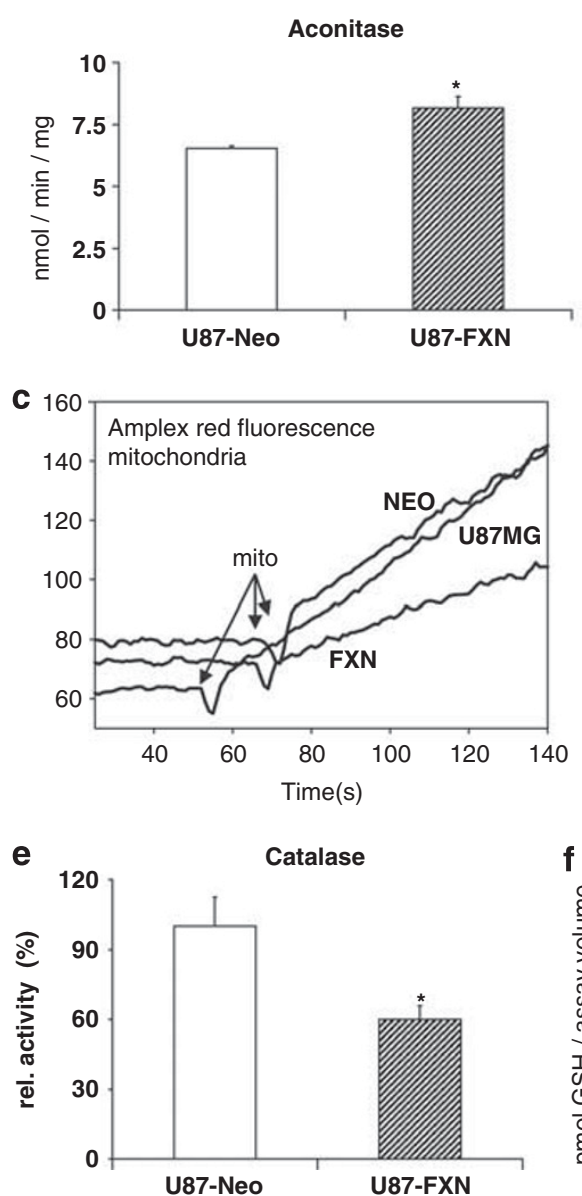

b

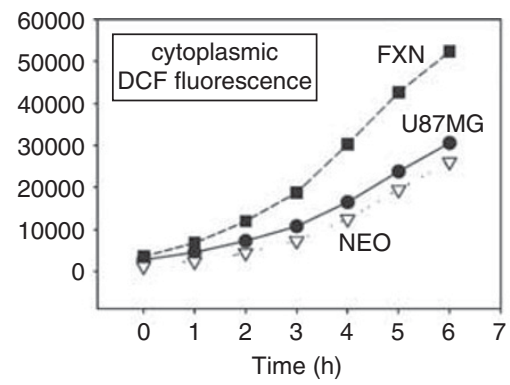

d

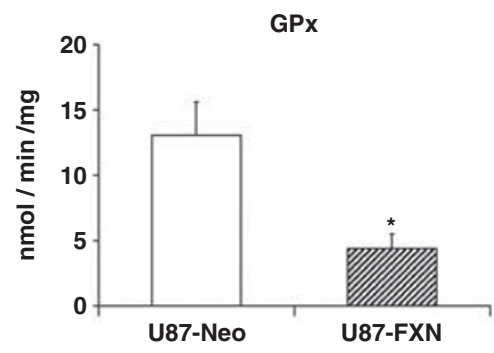

Glutathione

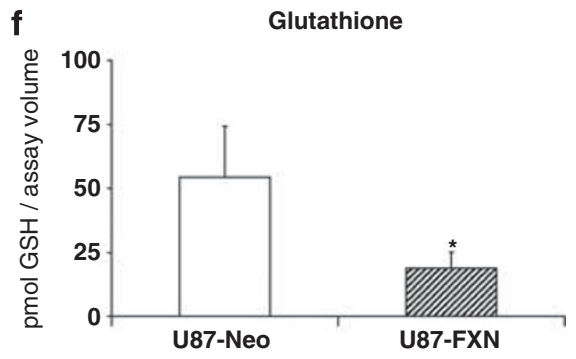

Figure 3 Effects of frataxin re-expression on ROS levels and antioxidative defence status in glioblastoma cells. (a) Increased aconitase activity in U87-FXN cells (six independent experiments, $P \leq 0.05$ ) indicates reduced mitochondrial oxidative stress. (b) Direct assessment of ROS production by measurement of DCF fluorescence reveals increased ROS levels in U87-FXN cells. The traces represent a single experiment chosen from a total of six independent experiments (cell culture passages), which all showed higher DCF signals as compared with both other cell lines, which had similar fluorescence. (c) Recording of the $\mathrm{H}_{2} \mathrm{O}_{2}$ release from isolated mitochondria, using the AR/HRP detection system. The shown traces of resorufin fluorescence are representative for a total of four independent experiments. The rates of $\mathrm{H}_{2} \mathrm{O}_{2}$ release calculated from the traces were 135 pmol $\mathrm{H}_{2} \mathrm{O}_{2} / \mathrm{min} / \mathrm{mg}$ of protein for U87-Neo, $156 \mathrm{pmol} / \mathrm{min} / \mathrm{mg}$ for U87MG and $74 \mathrm{pmol} / \mathrm{min} / \mathrm{mg}$ for U87-FXN cells. The arrows indicate the addition of mitochondria. (d-f) Determination of glutathione peroxidase activity (GPx, d), catalase activity (e) and glutathione (f) demonstrate reduced cytoplasmic antioxidative capacity in U87-FXN cells (six independent experiments, ${ }^{*} P \leq 0.05$ ).

Next, we analyzed antioxidant defences. Both $\mathrm{H}_{2} \mathrm{O}_{2}$-converting enzymes, GPx and CAT, showed a significant decrease in U87-FXN cells $(P<0.01)$ to values of 36.8 and $59.5 \%$, respectively (Figures $3 \mathrm{~d}$ and e). The GPx cofactor glutathione (GSH) was significantly diminished in U87-FXN cells as well (Figure 3f). This suggested a role of decreased $\mathrm{H}_{2} \mathrm{O}_{2}$ detoxification for the enhanced DCF and Amplex Red signals measured in these cells. No significant differences were observed with respect to total superoxide dismutase activity (data not shown).

The decreased total GSH content corresponded with a higher sensitivity of U87-FXN cells to the GSH-depleting compound buthionine sulfoximine (BSO) in MTT assays. Inhibition of GSH synthesis by BSO is expected to have a faster effect in cells with lower basal GSH levels. Following application of $1 \mathrm{mM}$ BSO for $24 \mathrm{~h}$, the percentage of viable cells in MTT assays was decreased to $44 \%$ in U87-FXN cells as compared with $81 \%$ in line U87-Neo cells (Figure 4a). Furthermore, the decrease of $\mathrm{H}_{2} \mathrm{O}_{2}$-detoxifying capacity explains a significantly increased sensitivity of U87-FXN cells toward high doses of externally applied $\mathrm{H}_{2} \mathrm{O}_{2}$, which was assessed in MTT assays $24 \mathrm{~h}$ after bolus application of 100 or $1000 \mu \mathrm{M} \mathrm{H}_{2} \mathrm{O}_{2}$ (Figure 4b). The addition of a moderate concentration of $10 \mu \mathrm{M} \mathrm{H}_{2} \mathrm{O}_{2}$ (data not shown) resulted in non-significantly decreased cell survival rates, which also did not differ between the two clones (93.4\% in U87-FXN cells vs $94.7 \%$ in U87-Neo). These data suggested that GBM cells containing frataxin are more susceptible to cellular stress induced by oxidants. 

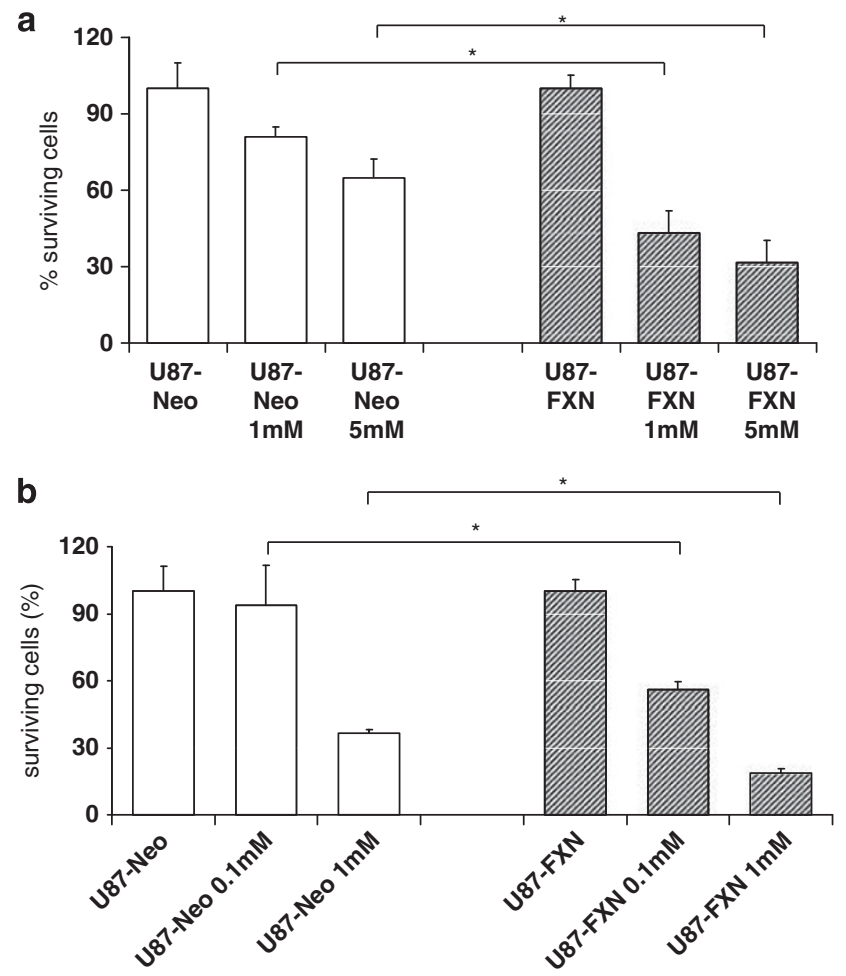

Figure 4 Increased susceptibility of frataxin-expressing glioma cells to external stressors. (a) Application of BSO (1 and $5 \mathrm{mM}$ ) which diminishes glutathione levels by blocking of de novo synthesis shows that U87-FXN cells are generally more susceptible than U87-Neo cells. (b) External application of hydrogen peroxide to glioma cells shows increased susceptibility of U87-FXN cells ( 4 independent experiments, 24 measurements per experiment, ${ }^{*} P \leq 0.05$ ).

\section{Growth Advantage of Frataxin-Containing Astrocytoma Cells}

BrdU assays repeatedly indicated a slightly higher rate of DNA synthesis for U87-FXN over U87-Neo cells in DMEM containing $10 \%$ FCS (data not shown). However, the difference reached statistical significance $(P \leq 0.001)$ only, if serum-starved cells were stimulated with 1\% FCS (Figure 5a). These results suggested a proliferative advantage for FXNoverexpressing cells under growth factor-restricted conditions. Tumor necrosis is a hallmark of GBM and a condition in which hypoxia and restricted supply with several growth factors occur. As shown in Figure 5b, the decrease of viable cells following hypoxic periods ( $0.1 \%$ oxygen) of 24 and $48 \mathrm{~h}$ was less pronounced in U87-FXN as compared with U87-Neo cells, as determined by MTT assay. This suggests a growth advantage of cells containing frataxin. To analyze tumor growth in vivo, we injected matrigel-embedded glioma cells subcutaneously into nude mice. Animals injected with U87FXN cells developed larger tumors than animals carrying U87-Neo cells. Weekly assessment of tumor volumes confirmed a significant growth advantage of U87-FXN cells (Figure 5c). U87-FXN tumors were highly necrotic, while U87-Neo tumors were characterized by a more solid growth (Figure 5d).

\section{Frataxin Enhances Sensitivity to Intrinsic Apoptosis Induction and Alkylating Drugs}

U87-FXN cells are more susceptible to external oxidative stress $\left(\mathrm{H}_{2} \mathrm{O}_{2}\right)$ than control cells, and, in general glioma cells exhibit a substantial resistance to apoptosis induction by chemotherapeutic drugs. ${ }^{19}$ Therefore, we wondered whether the FXN level of glioma cells might modulate apoptosis sensitivity and cytotoxic effects of alkylating agents currently used for glioma therapy.

First, we tested the sensitivity toward staurosporine, a wellknown trigger of intrinsic apoptosis. Staurosporine induced a significant cell loss within $24 \mathrm{~h}$. The dose response curve for U87-FXN cells was clearly below that one for U87-Neo cells over the whole range of applied concentrations (Figure 6a), suggesting a generally enhanced susceptibility of FXNexpressing cells to intrinsic apoptosis. Furthermore, cell survival after treatment with alkylating agents for $24 \mathrm{~h}$ was reduced in U87-FXN cells (Figure 6b). Cell loss was moderately reduced, if cells were pre-treated for $1 \mathrm{~h}$ with the antioxidants, included in the medium during BCNU treatment (Figure 6c). To further support a possible connection between BCNU sensitivity and oxidative stress, we analyzed, if BCNU leads to enhanced cytoplasmic ROS levels. A moderate increase of DCF fluorescence could be observed after $2 \mathrm{~h}$ of exposure to $20 \mu \mathrm{M}$ BCNU in U87-FXN cells (16.6\%) and U87-Neo cells (12.2\%).

MGMT $\left(0_{6}\right.$-methylguanine-DNA-methyltransferase $)$ is known to be expressed in a fraction of gliomas and to be crucial for the removal of alkyl residues from tumor DNA. It has been shown to be a major mechanism of resistance against alkylating drugs in GBM. The pattern of promoter methylation is known to possess significant predictive power regarding overall survival. Methylation analysis revealed a completely methylated status in both cell lines (data not shown), excluding a role of MGMT in the differential reaction to alkylating drugs.

\section{DISCUSSION}

Astrocytic FXN expression had been described only occasionally in the literature. ${ }^{15}$ Since we observed reduced FXN amounts in astrocytic tumor cells, preservation of normal astrocytic levels is not essential for tumor cell viability and proliferation. At first sight, this seems to be in contrast to results obtained recently by Guccini et al, ${ }^{20}$ who measured enhanced FXN expression in a series GBMs as compared with adjacent brain tissue. However, the extracts of tumor-free brain used by the authors as a reference, represent a mixture of cell types. The tissue may furthermore have been affected by peritumoral changes, such as edema. Since the authors used western blots of tissue homogenates, FXN upregulation cannot be clearly attributed to changes within the transformed astrocytes. On the other hand, comparisons of glioma cells with proliferating astrocyte cultures, as performed in the present study, solely represent an artificial approximation to the situation in vivo. It must be stated that no final decision 


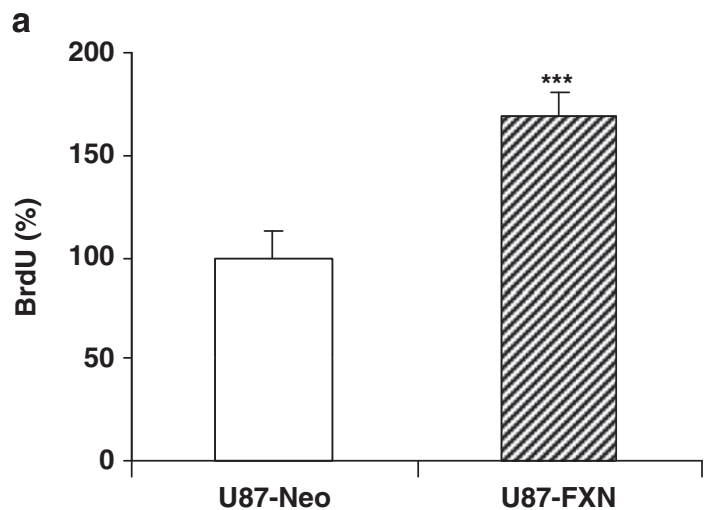

C

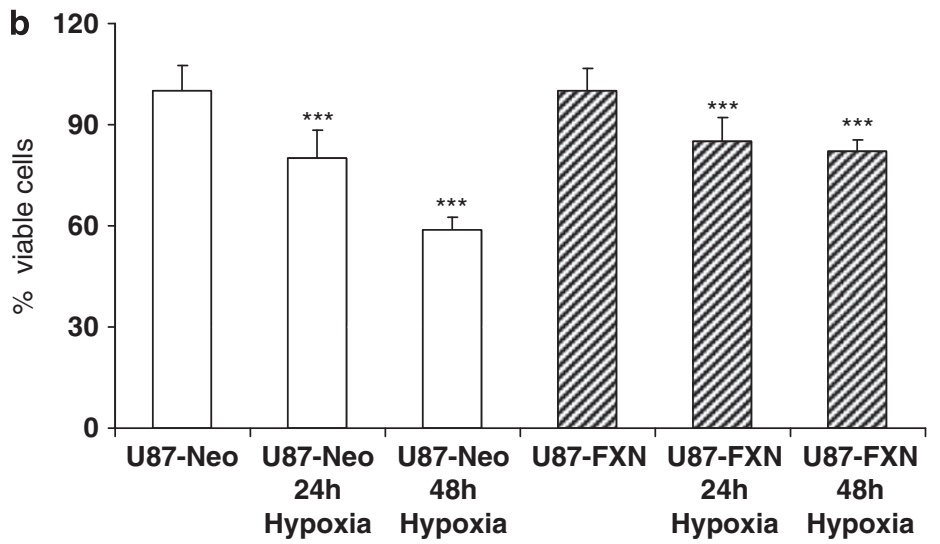

d

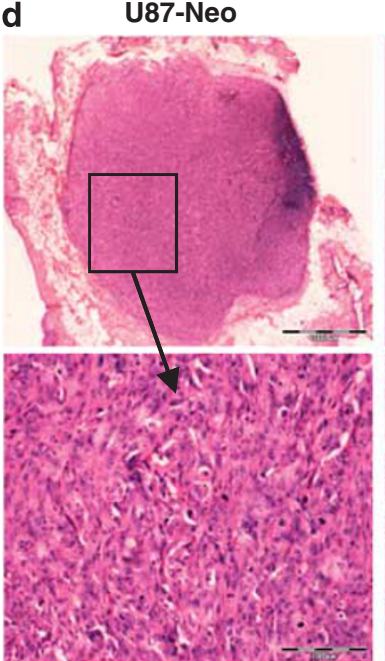

U87-FXN

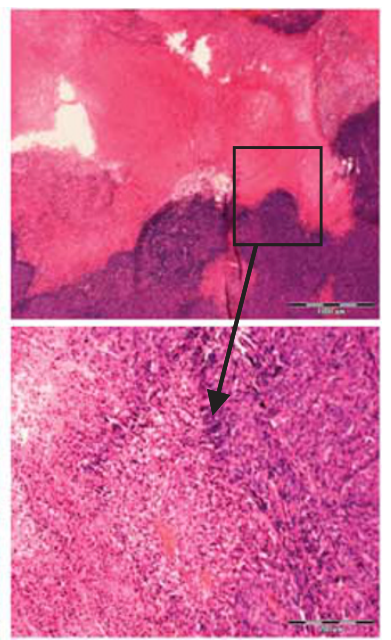

Figure 5 Cellular stress enhances cell survival and proliferation of frataxin-containing glioma cells. (a) If cells are grown under serum-restricted conditions (1\% serum), BrdU incorporation is significantly higher in U87-FXN cells ( ${ }^{* *} P \leq 0.001,4$ independent experiments, 24 measurements per experiment). (b) Cell culturing under hypoxic conditions renders U87-FXN cells less susceptible than U87-Neo control cells (4 independent experiments, 24 technical duplicates, ${ }^{* * *} P \leq 0.001$ ). The survival data for parental U87MG cells (data not shown) were not significantly different from those of U87-Neo cells, ie, $82.3 \%$ after $24 \mathrm{~h}$ and $62.8 \%$ after $48 \mathrm{~h}$ of hypoxia. (c) Growth of U87-FXN cells in nude mice reveals significantly increased tumor burden compared with controls $(* * P \leq 0.01)$. Two experiments with two passages of the cell clones had been performed. Each experiment contained four mice, subcutaneously injected with tumor cells on both sides. (d) Histological analysis of tumor xenografts shows largely necrotic tumors in mice injected with U87-FXN cells, while U87-Neo cells display solid growth without necroses.

can currently be made regarding the expression changes in astrocytes during tumor development.

We found evidence for a proliferation-supporting function of FXN under growth factor-restricted and hypoxic conditions, which may have a role in vivo depending on the microenvironment, such as supply with functional blood vessels. Guccini et $a l^{20}$ demonstrated a moderately enhanced FXN expression in various tumor cell types under hypoxia, including U87MG glioma cells (1.5-fold increase under $1 \%$ $\mathrm{O}_{2}$ ). These results may be interpreted in terms of a protective function of the mitochondrial protein under hypoxic conditions, which is in accordance with our observation of increased hypoxia survival of U87-FXN cells (Figure 5b).

Enhanced sensitivity of FXN-overexpressing cells to the apoptotic inducer staurosporine and to alkylating drugs points toward a potential role of FXN loss in the well-known apoptosis- and chemoresistance of astrocytic tumors. The result cannot be explained by enhanced apoptotic sensitivity of FXN transfected cells due to higher mitochondrial content, since CS activity was not altered by FXN transfection. Both enhanced apoptotic sensitivity and enhanced proliferation (as discussed below) may be explained by increased ROS levels, measured in U87-FXN cells. The nature of enhanced ROS levels remains unclear and may most likely be explained by the observed decrease of antioxidative defences. The cytoplasmic ROS increase was certainly not a consequence of an increased $\mathrm{H}_{2} \mathrm{O}_{2}$ release from mitochondria, since measurements in mitochondrial suspensions revealed lower ROS fluorescence. FXN seemed to protect mitochondria from ROS generation, as expected from the literature data for carcinoma cells. These results are in line with the moderately increased aconitase activity. 
a
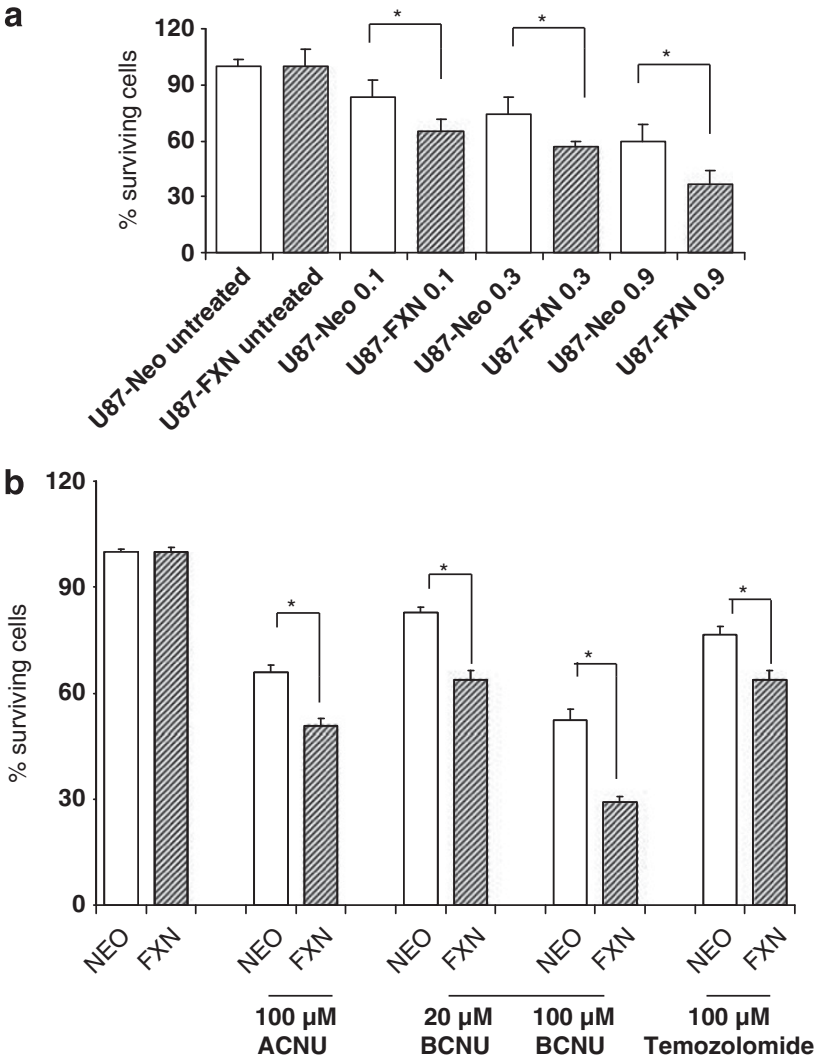

C

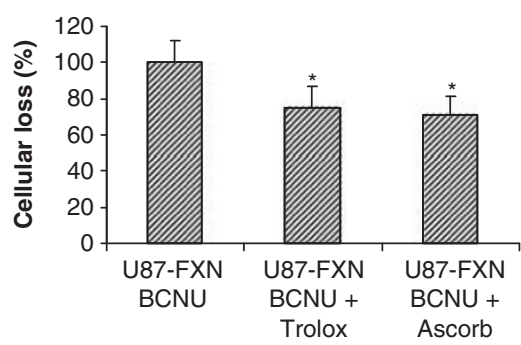

Figure 6 Increased susceptibility of frataxin-containing glioma cells to apoptotic inducers and alkylating drugs. (a) Cell survival assays show significantly reduced survival of U87-FXN cells following internal apoptosis induction by staurosporine. (b) Commonly used alkylating drugs for the treatment of gliomas are more effective in U87-FXN cells compared with control cells. (c) Effects of alkylating drugs (BCNU) can be partially rescued by concomitant application of antioxidative agents (trolox and ascorbate) in U87-FXN cells (4 independent experiments, 24 technical replications per experiment).

At this point, it remains unclear whether FXN regulates $\mathrm{H}_{2} \mathrm{O}_{2}$ generation at yet unknown sites of glioma cells, or whether solely a decrease of the hydrogen peroxide detoxifying enzymes catalase and glutathione peroxidase and GSH decline caused the enhanced cytoplasmic ROS levels in U87FXN cells. Independent of the precise mechanism, the enhanced sensitivity to cell death induction by external $\mathrm{H}_{2} \mathrm{O}_{2}$ and by BSO-induced GSH depletion, was in accordance with these data. It remains unclear, under which conditions FXN expression in eukaryotic cells either upregulates or downregulates ROS and antioxidative defences.
Taken together, the overexpression of FXN delivered inhomogeneous results in various eukaryotic cells. If an antioxidative function of the protein, as suggested in two carcinoma models ${ }^{9,10}$ is no general feature of tumor cells (present study), it is not acceptable to hypothesize that FXN generally acts as a tumor suppressor based on these antioxidative properties, as proposed earlier. ${ }^{10}$

Since FXN overexpression did not induce morphological changes and cells exhibited a high proliferation rate, it is not likely that the increased ROS levels cause 'oxidative stress' in an environment without additional noxes. The biological response of tumor cells to ROS is not always detrimental, but strongly depends on the concentration. Various tumor cell types, including astrocytoma cells, are known to increase proliferation in vitro following moderately increased ROS levels. ${ }^{21-23}$ More important, U87-FXN cells exhibited faster tumor growth in vivo, while FXN-induced ROS suppression was accompanied by suppressed tumor growth in vivo in the colorectal tumor model.

Pro-proliferative redox signaling has long been discussed in carcinogenesis, since several pathways downstream of receptor tyrosine kinases, such as mitogen-activated protein kinases, Akt and protein kinase C (PKC) can either be directly influenced by intracellular redox state or by the redox-sensitive transcription factor NFK-B. ${ }^{24}$ PKC may be regulated directly by ROS, since it contains cysteine residues, which may undergo ROS-induced redox modifications. ${ }^{25}$

Despite the above-discussed stimulating effects of moderately increased ROS levels, they may at the same time sensitize tumor cells to apoptotic triggers. Extrinsic and intrinsic (mitochondrial) apoptosis can be facilitated by ROS. ${ }^{26,27}$ DNA strand breaks and strand crosslinking induced by alkylating drugs also elicit intrinsic apoptosis. It is, therefore, not surprising to find an enhanced sensitivity of U87-FXN cells toward alkylating drugs, which was partially rescued by antioxidants. Enhanced sensitivity to chloroethylated nitrosoureas (such as ACNU and BCNU) may in addition be favored by the lowered GSH content of FXNexpressing cells, since GSH participates in detoxification of these drugs. ${ }^{28-30}$

Taken together, FXN may act as a double-edged sword in astrocytic tumors. This role may comprise a detrimental, growth promotion and, on the other hand, a beneficial chemosensitizing effect. Further studies are needed to delineate possible clinical relevance especially regarding chemoresistance evaluation.

\section{ACKNOWLEDGEMENTS}

We thank Michael Ristow for the human frataxin cDNA construct. ${ }^{31}$ The work was supported in part by a grant from the IZKF of the University of Jena (to $C M$ ). ML was supported by grants from the Wilhelm-SanderStiftung (2008-092-1) and the Deutsche Forschungsgemeinschaft. BK was supported in part by a Gerok position of the DFG (SFB 854). Frataxin research is further supported by research grants from the Deutsche Gesellschaft für Muskelkranke (DGM) and the Christa-Lorenz-Stiftung (to EK and CM). The skilful technical work of Ines Schellhase, Ines Meyer, 
Sandra Hartmann, Leona Buck and Heidelore Goldammer is highly appreciated. We also thank Dr Christine Richter-Landsberg for providing the OLN-93 cells. We also thank Dr Sanft from the Department of Forensic Science of the Friedrich-Schiller University Jena, Germany, for performing the cell line identification tests. We thank Dr S Krueger (Pathology, University of Magdeburg) for kindly performing densitometric scans.

\section{DISCLOSURE/CONFLICT OF INTEREST}

The authors declare no conflict of interest.

1. Calabrese V, Lodi R, Tonon $C$, et al. Oxidative stress, mitochondrial dysfunction and cellular stress response in Friedreich's ataxia. J Neurol Sci 2005;233:145-162.

2. Patel PI, Isaya G. Friedreich ataxia: from GAA triplet-repeat expansion to frataxin deficiency. Am J Hum Genet 2001;69:15-24.

3. Koutnikova $\mathrm{H}, \mathrm{Campuzano} V$, Foury $F$, et al. Studies of human, mouse and yeast homologues indicate a mitochondrial function for frataxin. Nat Genet 1997;16:345-351.

4. Babcock $M$, de Silva $D$, Oaks $R$, et al. Regulation of mitochondrial iron accumulation by Yfh1p, a putative homolog of frataxin. Science 1997;276:1709-1712.

5. Rotig A, de Lonlay P, Chretien D, et al. Aconitase and mitochondrial iron-sulphur protein deficiency in Friedreich ataxia. Nat Genet 1997;17:215-217.

6. Gakh O, Smith DY, Isaya G. Assembly of the iron-binding protein frataxin in Saccharomyces cerevisiae responds to dynamic changes in mitochondrial iron influx and stress level. J Biol Chem 2008;283: 31500-31510.

7. Leidgens S, De Smet S, Foury F. Frataxin interacts with Isu1 through a conserved tryptophan in its beta-sheet. Hum Mol Genet 2009;19: 276-286.

8. Bencze KZ, Yoon T, Millan-Pacheco $C$, et al. Human frataxin: iron and ferrochelatase binding surface. Chem Commun (Camb) 2007;14: $1798-1800$.

9. Schulz TJ, Thierbach R, Voigt A, et al. Induction of oxidative metabolism by mitochondrial frataxin inhibits cancer growth: Otto Warburg revisited. J Biol Chem 2006;281:977-981.

10. Thierbach $R$, Schulz TJ, Isken $F$, et al. Targeted disruption of hepatic frataxin expression causes impaired mitochondrial function, decreased life span and tumor growth in mice. Hum Mol Genet 2005;14: 3857-3864.

11. Furnari $F B$, Fenton $T$, Bachoo RM, et al. Malignant astrocytic glioma: genetics, biology, and paths to treatment. Genes Dev 2007:21:2683-2710.

12. Chamaon K, Kirches E, Kanakis D, et al. Regulation of the pituitary tumor transforming gene by insulin-like-growth factor-I and insulin differs between malignant and non-neoplastic astrocytes. Biochem Biophys Res Commun 2005;331:86-92.

13. Geserick P, Hupe M, Moulin M, et al. Cellular IAPs inhibit a cryptic CD95-induced cell death by limiting RIP1 kinase recruitment. J Cell Biol 2009;187:1037-1054.
14. Napoli E, Morin D, Bernhardt R, et al. Hemin rescues adrenodoxin, heme $a$ and cytochrome oxidase activity in frataxin-deficient oligodendroglioma cells. Biochim Biophys Acta 2007;1772:773-780.

15. Campuzano V, Montermini L, Molto MD, et al. Friedreich's ataxia: autosomal recessive disease caused by an intronic GAA triplet repeat expansion. Science 1996;271:1423-1427.

16. Gordon DM, Shi $Q$, Dancis $A$, et al. Maturation of frataxin within mammalian and yeast mitochondria: one-step processing by matrix processing peptidase. Hum Mol Genet 1999;8:2255-2262.

17. Schmucker $S$, Argentini $M$, Carelle-Calmels $N$, et al. The in vivo mitochondrial two-step maturation of human frataxin. Hum Mol Genet 2008;17:3521-3531.

18. Gardner PR, Fridovich I. Inactivation-reactivation of aconitase in Escherichia coli. A sensitive measure of superoxide radical. J Biol Chem 1992;267:8757-8763.

19. Sarkaria JN, Kitange GJ, James $C D$, et al. Mechanisms of chemoresistance to alkylating agents in malignant glioma. Clin Cancer Res 2008;14:2900-2908.

20. Guccini I, Serio D, Condo I, et al. Frataxin participates to the hypoxiainduced response in tumors. Cell Death Dis 2011;2:e123.

21. Arora-Kuruganti P, Lucchesi PA, Wurster RD. Proliferation of cultured human astrocytoma cells in response to an oxidant and antioxidant. J Neurooncol 1999;44:213-221.

22. Kuruganti PA, Wurster RD, Lucchesi PA. Mitogen activated protein kinase activation and oxidant signaling in astrocytoma cells. J Neurooncol 2002;56:109-117.

23. Martin V, Herrera F, Garcia-Santos G, et al. Signaling pathways involved in antioxidant control of glioma cell proliferation. Free Radic Biol Med 2007:42:1715-1722.

24. Valko M, Rhodes CJ, Moncol J, et al. Free radicals, metals and antioxidants in oxidative stress-induced cancer. Chem Biol Interact 2006;160:1-40

25. Gopalakrishna R, Gundimeda U. Antioxidant regulation of protein kinase $C$ in cancer prevention. J Nutr 2002;132:3819S-3823S.

26. Ryter SW, Kim HP, Hoetzel A, et al. Mechanisms of cell death in oxidative stress. Antioxid Redox Signal 2007;9:49-89.

27. Circu ML, Aw TY. Reactive oxygen species, cellular redox systems, and apoptosis. Free Radic Biol Med 2010;48:749-762.

28. Ali-Osman F, Caughlan J, Gray GS. Decreased DNA interstrand crosslinking and cytotoxicity induced in human brain tumor cells by 1,3bis(2-chloroethyl)-1-nitrosourea after in vitro reaction with glutathione. Cancer Res 1989;49:5954-5958.

29. Weber GF, Waxman DJ. Denitrosation of the anti-cancer drug 1,3bis(2-chloroethyl)-1-nitrosourea catalyzed by microsomal glutathione S-transferase and cytochrome P450 monooxygenases. Arch Biochem Biophys 1993;307:369-378.

30. Ali-Osman F, Stein DE, Renwick A. Glutathione content and glutathione-S-transferase expression in 1,3-bis(2-chloroethyl)-1nitrosourea-resistant human malignant astrocytoma cell lines. Cancer Res 1990;50:6976-6980.

31. Shoichet SA, Baumer AT, Stamenkovic D, et al. Frataxin promotes antioxidant defense in a thiol-dependent manner resulting in diminished malignant transformation in vitro. Hum Mol Genet 2002;11:815-821. 